\title{
Accurate Tongue-Palate Pressure Sensing Device to Study Speech Production and Swallowing in Patients with Complete Denture
}

\author{
Bharat Mirchandani ${ }^{1,2} \quad$ Pascal Perrier $^{1} \quad$ Brigitte Grosgogeat ${ }^{2,3,4} \quad$ Christophe Jeannin ${ }^{1,2,3,4, \odot}$ \\ 1Université Grenoble Alpes, CNRS, Grenoble INP, GIPSA-Lab, \\ Grenoble, France \\ 2Laboratoire des Multimatériaux et Interfaces, UMR CNRS Université \\ de Lyon, Université Claude Bernard Lyon 1, France \\ Address for correspondence Christophe Jeannin, DDS, LMI - UMR \\ CNRS 5615, Université de Lyon, Université Claude Bernard Lyon1, \\ Faculté d'odontologie, 11, rue Guillaume Paradin, 69372 \\ Lyon 08, France (e-mail: christophe.jeannin@univ-lyon1.fr).
}

${ }^{3}$ Hospices Civils de Lyon, Lyon, France

${ }^{4}$ Faculté d'odontologie de Lyon, Lyon, France

Eur J Dent 2021;15:302-306

\begin{abstract}
Keywords

- tongue-palate interactions

- complete denture

- speech production

- swallowing

- strain-gauge sensors

Objectives The mechanical interactions between tongue and palate are crucial for speech production and swallowing. In this study, we presented examples of pressure signals that can be recorded with our PRESLA system (PRESLA holds for the French expression "PRESsion de la LAngue" [Pressure from the tongue]) to assess these motor functions, and we illustrate which issues can be tackled with such a system.

Materials and Methods A single French-speaking edentulous subject, old wearer of a complete denture, with no speech production and swallowing disorders, was recorded during the production of nonsense words including French alveolar fricatives, and during dry and water swallowing. The PRESLA system used strain-gauge transducers that were inserted into holes drilled in the palatal surface of a duplicate of the prosthesis at six locations that were relevant for speech production and swallowing. Pressure signals were postsynchronized with the motor tasks based on audio signals.

Results Patterns of temporal variations of the pressure exerted by the tongue on the palate are shown for the two studied motor tasks. It is shown for our single subject that patterns for fricative /s/are essentially bell shaped, whereas pressure signals observed for water swallow begin with a maximum followed by a slow decrease during the rest of the positive pressure phase. Pressure magnitude is almost 20 times larger for water swallow than for $/ \mathrm{s} /$ production.

Conclusions This study illustrates the usefulness of our PRESLA system for studying speech production and swallowing motor control under normal and pathological conditions.
\end{abstract}

\section{Introduction}

To study with good spatial and temporal accuracies the mechanical interactions between tongue and palate in speech production and swallowing, we have developed a system called PRESLA, ${ }^{\text {a }}$ which relies on the use of a palatal plate

${ }^{\text {a }}$ PRESLA holds for the French expression "PRESsion de la LAngue" (Pressure from the tongue).

published online January 7, 2021
DOI https://doi.org/

$10.1055 / \mathrm{s}-0040-1717002$ ISSN 1305-7456. with embedded strain-gauge sensors and innovates by using complete edentulous subjects ${ }^{1}$ who have been using their complete denture for months and adapted to articulate and swallow with it. ${ }^{2}$ In the recent years, the PRESLA system beneficiated from numerous improvements, including the design of specific acquisition chain, which enables high signal-to-noise ratio, an increased number of sensors, specific data-processing programs, and an improvement in the calibration device. This

(c) 2021. European Journal of Dentistry.

This is an open access article published by Thieme under the terms of the Creative Commons Attribution-NonDerivative-NonCommercial-License, permitting copying and reproduction so long as the original work is given appropriate credit. Contents may not be used for commercial purposes, or adapted, remixed, transformed or built upon. (https://creativecommons.org/licenses/by-nc-nd/4.0/)

Thieme Medical and Scientific Publishers Pvt. Ltd., A-12, 2nd Floor, Sector 2, Noida-201301 UP, India 
report briefly documents the technical aspects of our experimental setup, and presents first results for fricative consonants and swallowing experiments obtained from a single subject.

\section{Materials and Methods}

\section{Participant}

A 54-year-old completely edentulous male subject, native French speaker, served as subject. He received all the required information about the experimental setup, the protocol, and our research aims, and signed informed consent. His participation is part of the pilot study associated with the ethical committee application 69HCL18_0103 promoted by the University Hospital of Lyon.

The subject had worn maxillary and mandibular complete denture satisfactorily for 3 years. An important issue in edentulous patients, which has been frequently addressed in the literature, is the so-called "macroglossy," a term abusively used to refer to the apparent increase in the volume of the tongue in its anterior part. It actually refers to the fact that in these patients the shape of the tongue is modified due to the absence of teeth: the tongue spreads over the edentulous ridges, which induces a reduction in the vertical dimension of the air channel and limits the freedom of the tongue to move vertically. Our subject did not suffer from this problem. The clinical evaluation of his oral cavity has revealed that by the time of the experiment he had recovered nonpathological physiological parameters, in particular with a normal size of the air channel between the tongue surface and the palatal vault, mainly due to the correct restoration of the vertical dimension of occlusion and to an appropriate thickness of the prosthesis, which preserves the freedom of the tongue to move vertically and horizontally in the vocal tract. In addition, informal evaluations of his speech production did not present any indications of speech disorders. In agreement with these observations, this subject reported no history of medical conditions such as speech impairments and dysphagia. He had also no history of hearing disorders. Hence, we consider that our subject had fully adapted to his complete prosthesis and had normal speech production and swallowing by the time of the experiment. Importantly, we consider then that the data collected from this subject inform about normal speech production and swallowing.

\section{Subject-Specific PRESLA System}

The original PRESLA system has been presented in our seminal paper published in Material Science and Engineering $C .{ }^{1}$ In particular, details about the strain-gauge sensors and the instrumentation tools can be found in Figure and sections 2.1.4 and 2.1.5 of this seminal paper (pages 836-837). Additional information about the recent improvements in the setup is given in our recent paper $^{3}$ published in Clinical Linguistics and Phonetics, in section "Material," pages 57-61. We focus here on experimental aspects associated with the investigation of speech production and swallowing in our subject.

Six strain-gauge transducers were inserted into the palatal surface of the duplicate ( $\mathbf{- F i g . 1 B}$ ). To determine the locations

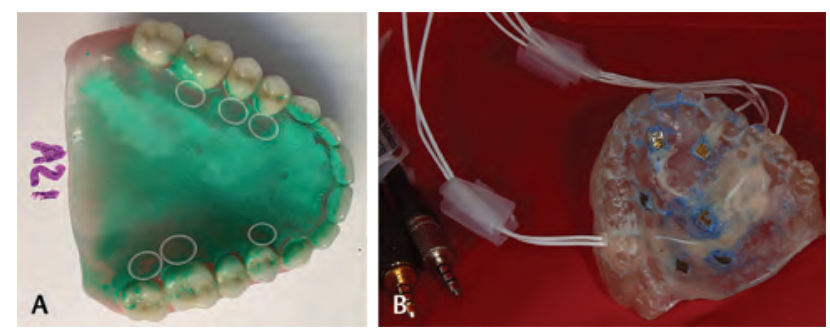

Fig. 1 (A) Palatogram for fricative consonant /s/ on existing complete denture to determine sensor position. (B) Sensors placed into the duplicate. The wires are placed inside the thickness of the duplicate. Sensor surfaces flush with the palatal plate.

of sensors in the palatal plate, high-quality green spray (Occlu Spray Plus, Hager \& Werken GmbH \& Co. KG, Germany) palatograms were done on an accurate duplicate of the subject's complete denture, for both speech and swallowing tasks (one recording per swallowing task and per consonant; - Fig. 1A).

Small holes were drilled in the palatal plate to accommodate the sensors, without altering the palatal vault of the complete denture. Each sensor was fixed on a plastic disk to avoid the effect of strain in the resin, taking care to ensure the free movement of the strain gauge. The sensing surface was aligned along with the palatal surface. The wires connecting the sensors to the amplifier, placed within the thickness of the base plate, exit from the vestibular side of the premolar area by the way of labial commissure.

\section{Calibration}

Calibration of all the sensors was performed in a specially constructed setup we have developed to have conditions similar to those of the experiment, especially as regards the soft characteristics of tongue tissue, to measure tongue pressure. The calibration setup is based on the concept of water column exerting a perfectly controlled pressure on the sensor via a latex finger ( $\boldsymbol{- \text { Fig. }}$ 2). The experimental denture was firmly mounted on a fixed vice with a height and an inclination enabling that the latex contacted the sensor at essentially right angle and without exerting any stress on it. This configuration was considered to determine the reference water height and reference sensor output associated with a zero-mechanical pressure on the sensor. Mechanical pressure was then applied to the sensor by increasing precisely the water height. The relation between the mechanical pressure and the output voltage of the electronic measurement system (a Wheatstone bridge) has been shown to be linear in the range [0, $5 \mathrm{kPa}$, and we assumed this linear relation to apply over a larger range up to $50 \mathrm{kPa}$ (see Mirchandani et al ${ }^{3}$ for details).

The accuracy of the pressure measurement provided by PRESLA was evaluated with the water column across five repetitions of the measure of the voltage induced in a sensor for controlled pressures varying in the range $1.5-4 \mathrm{kPa}$. We found a variation across repetitions smaller than $2 \%$, which corresponds to a very good accuracy.

\section{Data Collection}

The data from the six sensors were gathered simultaneously via a low-noise amplifier, especially made for this 

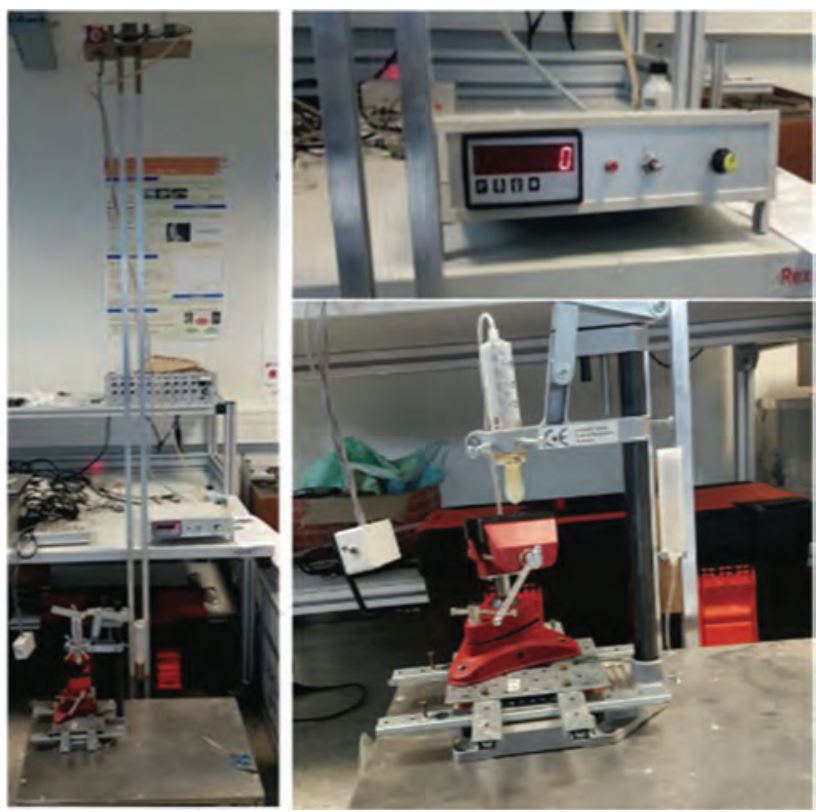

Fig. 2 Global view of the calibration device (left); latex finger and vice holding the denture (right bottom); and electric motor display showing the height of the water (right top).

research (sampling frequency: $4 \mathrm{kHz}$ ). Speech sounds were recorded via a microphone with a multichannel audio recorder (M-Audio Fast Track Pro) using Audacity software at $22.05 \mathrm{kHz}$. During the swallowing tasks the patient was asked to manually push on a beep button, to signal his swallowing onset and offset. The beep was loudly played in the room via a loudspeaker so that it could be recorded with the microphone, with the same procedure as for the speech sounds. Pressure and audio signals (speech and beeps) were a posteriori synchronized thanks to a square signal that was sent simultaneously to the low-noise amplifier and to the audio recorder. The data acquisition was made by MATLAB software and the data were displayed online as time-varying signals on the laptop screen.

During the recording, the subject was seated in a dental chair in an upright position with the head stabilized by the headrest. For the speech production task described in this paper, the subject was instructed to pronounce repetitions of two [i]C[a] and two [a]C[i] nonsense words, where $\mathrm{C}$ was a French fricative consonant among $\mid \mathrm{s} /$ and $|\mathrm{z}|$ in different random order; /isa/ /asi/ /iza/ /azi/. The swallowing tasks consisted of swallowing of saliva and $10 \mathrm{~mL}$ of water. Ten measurements were taken for each task.

\section{Results}

Examples of recorded data from the sensor located in the alveolar region of the PRESLA system are shown in - Fig. 3, which show typical pressure patterns observed for the articulation of unvoiced fricative /s/ in /isa/ (top panel), and for swallowing (bottom panel).

Relevant time events for the production of $/ \mathrm{s} /$, labeled using the spectrotemporal characteristics of the speech

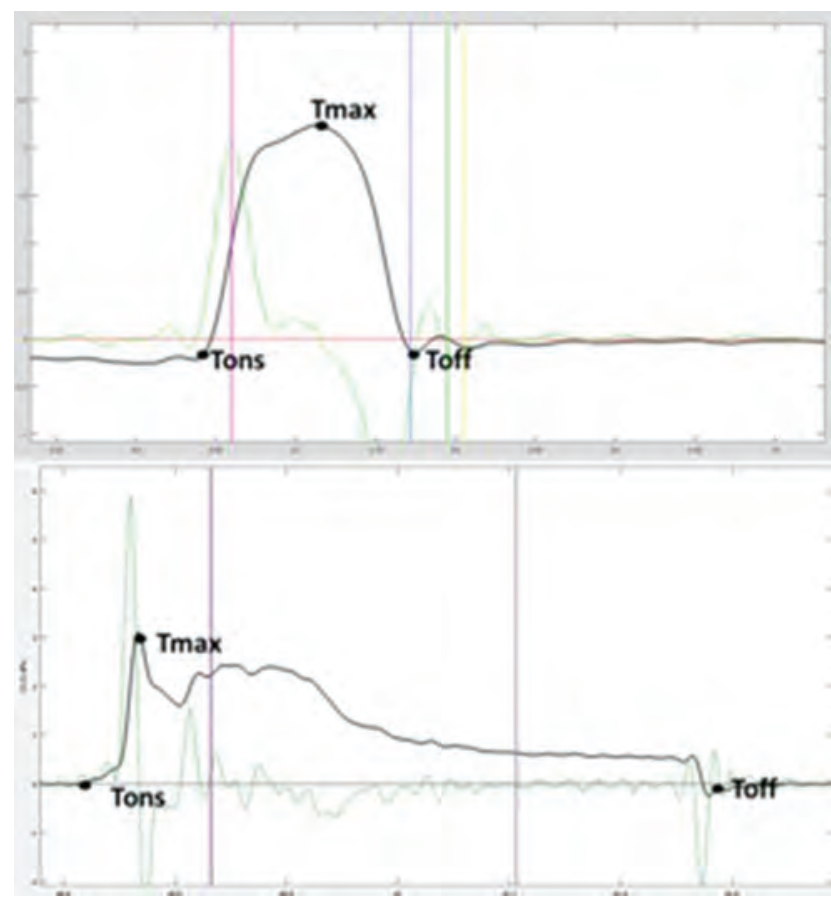

Fig. 3 Typical pressure signals measured with a sensor in the alveolar region of the PRESLA system. Top panel: speech production, /s/ in /isa/; the vertical colored lines represent acoustic time events; magenta $=$ onset of consonantal noise, blue $=$ offset of consonantal noise, green $=$ onset of postconsonantal vocal fold vibration, and yellow = onset of postconsonantal laminar airflow. Bottom panel: swallowing, water swallow; the two vertical lines mark the onset and offset times of swallowing as indicated by the subject with the beeps. In both panels: $\mathrm{T}_{\text {ons }}=$ onset time of positive pressure phase; $\mathrm{T}_{\text {off }}=$ offset time of positive pressure phase; $\mathrm{T}_{\max }=$ time of the maximum of the positive pressure phase. The green curve denotes the first time-derivative of the pressure signal.

signal, are indicated in the top panel with vertical lines. We observe that the onset of mechanical pressure precedes the onset of the consonantal noise. Hence, the noise does not start when tongue starts to be in contact with the palate, but when the area of the vocal tract constriction is small enough (below $30 \mathrm{~mm}^{2}$ according to Stevens, ${ }^{4}$ p. 1188) to significantly increase the air pressure in the back cavity of the vocal tract and generate turbulences in the constriction. Interestingly tongue pressure further increases after the consonantal noise onset, reaches a maximum, and immediately decreases after it. The offset of the consonantal noise occurs after the release of the mechanical pressure against the palate, which shows that air pressure does not instantaneously decrease.

For water swallow (bottom panel) the mechanical pressure reaches its maximum in the very first part of the positive pressure phase, and regularly decreases in the second half, which is consistent with the fact that swallowing starts with a sealing of the vocal tract in its anterior part where the observed sensor is located. Interestingly, the subject reported the onset of his swallow (first vertical line on the figure) almost $200 \mathrm{~ms}$ after the onset of positive tongue-palate pressure in the alveolar region. This suggests that the representation of the swallowing task for the subject could be more associated with its pharyngeal phase than with its palatal phase. 
To assess the general nature of the time variations depicted in - Fig. 3, we have provided for each task a statistical characterization of the pressure signal. To do so, we have first time-normalized the pressure signals by dividing the time by the duration of the positive pressure phase

$$
\left(t_{\text {normalized }}=\frac{t-T_{\text {ons }}}{T_{\text {off }}-T_{\text {ons }}}\right)
$$

and we have then plotted the 95\% confidence interval and the prediction interval at one standard deviation of the time-normalized signals recorded for all the repetitions of the same task. The 95\% confidence interval corresponds at each normalized time to the range of values in which the average value of the considered signal should be with a 0.95 probability. The prediction interval refers for each normalized time to the range of values that is likely to include the value of the measured signal for any single new observation. Hence the prediction interval reflects at each normalized time the uncertainty on the value of each single observation of the considered signal, while the confidence interval reflects at each normalized time the uncertainty around the mean value of the considered variable. - Figure 4 shows the results obtained for the repetitions of /s/ in /isa/ (Panel A) and water swallow (Panel B). It can be observed that, consistent with the single observation of $\mathbf{-}$ Fig. $\mathbf{3}$, pressure signals for /s/ are essentially bell shaped, with the decreasing phase starting immediately after the end of the rising phase, whereas pressure signals observed for water swallow begin with a maximum followed by a slow decrease during the rest of the positive pressure phase. Moreover, we observed that the pressure magnitude is almost 20 times larger for water swallow than for $/ \mathrm{s} /$ production. All these observations are consistent with basic knowledge of the motor control mechanisms underlying each task: in swallowing, tongue-palate contacts in the front part of the vocal tract must be strong enough to provide a true sealing of the vocal tract and the tongue takes advantage of these contacts to initiate its backward undulatory movement, which propels the bolus toward the pharynx; in /s/ production, the tongue gently touches the palate to stabilize the tongue and generate the small vocal
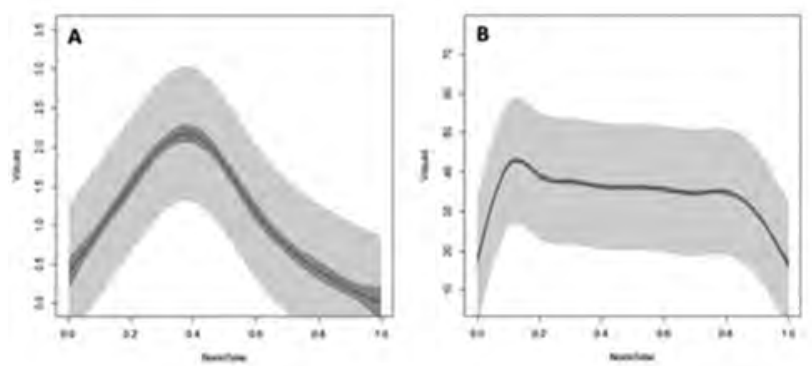

Fig. 4 Statistical distributions of the tongue-palate mechanical pressure for fricative /s/ in /isa/ (A) and water swallowing (B) for a sensor located in the alveolar region (front part of the complete denture). Pressure time patterns normalized in time by the whole duration of the positive pressure phase (see text for details). Dark gray shaded region: 95\% confidence interval; light gray shaded region: prediction interval at one standard deviation. tract constriction that gives birth to air turbulences and to the consonantal noise.

\section{Discussion}

Providing a precise description of the time variation of tongue-palate pressure in speech production and swallowing should help formulate strong hypotheses about the nature of motor control underlying the correct achievement of the tested motor tasks. In a recently published paper, ${ }^{3}$ we have shown that the production of the French velar stop / $\mathrm{k} /$ is associated with a plateau-like pattern of tongue pressure variation during vocal tract occlusion. We have interpreted the plateau-like pattern as evidence for the hypothesis that the production of velar stops is associated with tongue movement toward a virtual target located above the palate, a target whose virtual achievement would be marked by the pressure plateau. We have found above that the French fricative /s/ does not show such a plateau-like pressure pattern, but rather a bell-shaped pattern with a significantly smaller magnitude, suggesting a more gradual and less strong interaction between the tongue and the palate. This could reflect a complex interplay between the tongue, the palate, and the airflow going through the small constriction of the vocal tract in the palate-alveolar region (see for example Perrier et al, 2000, for a modeling of such an interplay). ${ }^{5}$ We also observed a significantly different pressure pattern for water swallow, which we could interpret in relation with the sealing of the vocal tract and the undulatory movement of the tongue required in this task.

Our results confirm that the PRESLA system and its associated experimental protocol involving complete denture wearers is well suited to the study of tongue-palate interactions in speech production and swallowing. This can be done in old wearers of their prosthesis, to study nonpathological condition, or with new wearers, to investigate adaptation mechanisms. Obviously, further investigations are required to further pursue the issues raised above. There are indeed obvious limitations in our study. First, data were collected from a single subject. Inter-subject variability is a wellknown characteristic of oral motor functions, in particular speech production, and extending this experimental protocol to a large number of subjects (classically 20-30 in speech production) is a requirement, to draw general conclusions about speech and swallowing motor control under normal conditions. Second, the calibration system of the sensors would beneficiate from improvements that would enable pressure measurements over a range larger than 0-5 kPa, to validate our assumption of a linear voltage-pressure relation for the whole domain of variation of the tongue-palate pressure. This is particularly important for swallowing, in which pressure can be larger than $50 \mathrm{kPa}$. We are also working on an improvement in the experimental protocol for swallowing. Currently, the postsynchronization of the pressure signals with the actual motor task is based on the audio signal that the subject has generated, when he was considering that he was swallowing. This induces a temporal incertitude, due to the subjective evaluation by 
the subject of the onset of his swallowing gesture and to the variability in the time interval between this subjective onset and the actual hand gesture that pushes the beep button. Improvements involve video recording of the neck region, to detect the onset of laryngeal elevation.

\section{Conclusions}

Using the temporal information of our recordings, patient can be taught to position the tongue in purposeful manner to improve retention and stability of denture. Constant repetition with use of coordinated exercises can help these patients learn muscular activity patterns to aid in retention and stability. ${ }^{6}$ For these reasons, the capability of our experimental device to record mechanical interactions at multiple sites of prosthesis is an important step toward the specification of general principles for the design of complete denture, to make most of the tongue support and facilitate the good quality of speech and swallowing. These goals if achieved will improve the comfort of edentulous patients.

Our device can also be useful together with other clinical evaluation to quantitatively assess the impact of orofacial disorders such as myofascial pain, ${ }^{7}$ maxillary odontogenic myxoma, ${ }^{8}$ or associated with maxillofacial prosthetic obturator. $^{9}$

\section{Funding}

This work was supported by the ARC2 grant (AuvergneRhône-Alpes region) under Grant 19691774400019, the Fondation des Gueules Cassées for study material and clinical research, and Doerler Mesures company that contributed to the cost of the transducers' production.

\section{Conflict of Interest}

None declared.

\section{References}

1 Jeannin C, Perrier P, Payan Y, Dittmar A, Grosgogeat B. Tongue pressure recordings during speech using complete denture. Mater Sci Eng C 2008;28:835-841

2 Petrović A. Speech sound distortions caused by changes in complete denture morphology. J Oral Rehabil 1985;12(1):69-79

3 Mirchandani B, Perrier P, Grosgogeat B, Jeannin C. Study of tongue-palate pressure patterns during the hold phase in the production of French denti-alveolar and velar stops. Clin Linguist Phon 2020;34(1-2):54-71

4 Stevens KN. Airflow and turbulence noise for fricative and stop consonants: static considerations. J Acoust Soc Am 1971;50(4B):1180-1192

5 Perrier P, Payan Y, Perkell J, et al. An attempt to simulate fluidwalls interactions during velar stops, Proceedings of the 5th Speech Production Seminar and CREST Workshop on Models of Speech Production. Kloster See on Bavaria, Germany; 2000:149-152

6 Brill N, Tryde G, Schübeler S. The role of learning in denture retention. J Prosthet Dent 1960;10:468-475

7 Sener S, Akgunlu F. Sociodemographic comparison in patients with subjective and objective clinical findings of temporomandibular dysfunctions. Eur J Dent 2011;5(4):380-386.

8 Rashid H, Bashir A. Surgical and prosthetic management of maxillary odontogenic myxoma. Eur J Dent 2015;9(2):277-283

9 Murat S, Gurbuz A, Isayev A, Dokmez B, Cetin U. Enhanced retention of a maxillofacial prosthetic obturator using precision attachments: two case reports. Eur J Dent 2012;6(2):212-217 